\title{
Arne Heise
}

\section{Postkeynesianische Finanzpolitik zwischen Gestaltungsoptionen und Steuerungsgrenzen}

\section{Von der finanzpolitischen Diskussion zur Pensée unique}

Die öffentliche Haushaltspolitik (Finanz- und Fiskalpolitik) gehört zu den umstrittensten Arenen der politischen wie der wissenschaftlichen Kontroverse. Dies kann natürlich nicht verwundern: Staatliche Aktivität - zumindest wenn es sich um prozeßpolitische Interventionen handelt - muß ihren Niederschlag in den Finanzplänen der öffentlichen Körperschaften finden. Die öffentliche Haushaltspolitik ist deshalb der monetäre Spiegel der jeweiligen politischen Richtungen und Ideologien, die den Staat führen (vgl. Schumpeter 1918). Kontroversen hierüber gehören zur demokratischen Kultur und sind Teil des Wahlaktes in repräsentativen Demokratien. Gleichermaßen zwangsläufig scheinen unterschiedliche wissenschaftliche Standpunkte zur Finanzpolitik, wenn wir uns vergegenwärtigen, dass es sich bei der Ökonomie um eine Disziplin mit mehreren Paradigmen handelt: Etwas grobgliederig können wir zwischen den walrasianischen Theorien mit ihrem am allgemeinen Gleichgewicht orientierten Selbststeuerungsoptimismus und den nichtwalrasianischen, heterodoxen Theorien mit ihrem Festhalten an der inhärenten Krisenanfälligkeit des Kapitalismus unterscheiden (vgl. z.B. Gerrard 1996). Unabhängig von der Herleitung staatlicher Funktionen und der normativen Festsetzung der Bereitstellung öffentlicher Güter muß es zwischen den beiden genannten Paradigmen selbst dann zu kontroversen Auffassungen kommen, wenn man sich auf prozeßpolitische Eingriffe angesichts von "Marktfehlern beschränken würde. Über dieses Schisma wird - in der gebotenen Kürze später noch zu sprechen sein.

Vor diesem Hintergrund muß es allerdings unverständlich erscheinen, dass es gegenwärtig weder in Politik noch Wissenschaft heftige Auseinandersetzungen

Eine frühere Version dieses Papers wurde auf der Arbeitstagung 'Makroökonomie, Beschäftigung und Nachhaltigkeit' vorgetragen, die am 13. Oktober 2000 in Berlin stattfand. Der Autor schuldet den Teilnehmern dieser Tagung, insbesondere Elmar Altvater, Ingo Schmidt und Axel Troost, Dank für kritische Kommentare, die zu mancher Präzisierung zwangen. 
um den richtigen Weg in der Finanzpolitik gibt - im Gegenteil: Es herrscht eine Einmütigkeit hinsichtlich der Konsoldierungsnotwendigkeiten wie nie zuvor. Im globalen Kapitalismus hat sich der ,Washington Consensus' (vgl. Matzner 2000) ,gesunder öffentlicher Finanzen' durchgesetzt, in der Europäischen Union die im Stabilitäts- und Wachstumspakt festgelegte restriktive Auslegung der Maastrichter Konvergenzkriterien (vgl. Arestis/ McCauley/Sawyer 2001). In der Bundesrepublik gibt es eine parteiübergreifende Übereinstimmung, dass nur ,Sparhaushalte Bestandteil guter, moderner Wirtschaftspolitik sein können. Mit ihrer ,TINA-Rhetorik' (There is no alternative) ist es Bundeskanzler Schröder und Finanzminister Eichel gelungen, auch einer, linken“ Partei im vormals ideologisch gespaltenen Wählerspektrum eine Finanzpolitik zu verabreichen, die schwerlich den Interessen ihrer Wählerschaft dient (vgl. Heise 1999 und dazu später mehr). Und in der Wissenschaft werden längst nur mehr die Risiken und Beschränkungen der staatlichen Finanzpolitik diskutiert, nicht mehr die Chancen und Möglichkeiten einer offensiv gestalteten Haushaltspolitik (vgl. z.B. Schlesinger/Weber/Ziebarth 1993).

Bevor im nächsten Abschnitt das paradigmatische Schisma zwischen (neo-) walrasianischer und (post-)keynesianischer Finanzpolitik diskutiert wird, muß kurz auf das finanzpolitische Gebahren des staatlichen Akteurs in der Bundesrepublik während der letzten drei Jahrzehnte eingegangen werden, denn es spannt den Wahrnehmungsrahmen auf, der für die aktuelle Konsolidierungsdiskussion und die TINA-Rhetorik maßgeblich ist. In der Entwicklung von der keynesianisch inspirierten Politik der Globalsteuerung der 1970er Jahre zur neoliberalen Politik des Nulldefizits der späten 1990er Jahre sind drei Phasen zu unterscheiden:

Phase I (1970er Jahre): In dieser Phase kommt es durch den säkularen Fall der Wachstumsrate des BIP auf ein langfristiges Niveau von real etwa 2\% (vgl. Lindlar 1997) und den gegenläufigen Anstieg des Zinsniveaus der öffentlichen Schuld auf real 2 - 4\% einerseits zur Domar'schen Verschuldungfalle ${ }^{1}$, andererseits fällt die Investitions- bzw. Akkumulationsrate unter ihr Vollbeschäftigungsniveau und es entsteht dauerhafte Unterbeschäftigung. Die ZinsWachstums-Differenz allein bewirkt allerdings nur ein langsames Anwachsen der öffentlichen Verschuldung und Zinslast.

Phase II (1980er Jahre): In dieser Phase reagieren Geld- und Finanzpolitik restriktiv auf die Entwicklungen der Phase I, denn auf die wachsende Verschuldung wird mit dem ,Konsolidierungsreflex' geantwortet und die Notenbank sieht sich genötigt, durch geldpolitische Restriktionen jede Hoffnung - oder

1 Die Domar'sche Verschuldungsfalle tut sich immer dann auf, wenn der Zinssatz der öffentlichen Schuldpapiere über der Rate des Wirtschaftswachstums liegt - dann nämlich steigt die öffentliche Schuldenquote, wenn die laufenden Haushalte nicht (zinsbereinigte) Primärüberschüsse bilden. 
Befürchtung - dass ein monetärer Bail-out (d.h. eine „Inflationssteuer“) eintreten könne, zunichte zu machen. ${ }^{2}$ Als Folge gerät die Akkumulationsmaschine des Kapitalismus weiter ins Stottern, aber auch die Domar'sche Schere geht noch weiter auseinander und ermöglicht trotz hoher Primärüberschüsse und einer deutlichen Kürzung der strukturellen Netto-Neuverschuldung den Insignien der Konsolidierung - keine Rückführung der Schuldenquote, da die gesamte Netto-Neuverschuldung als kreislauftheoretisches Residuum weiterhin hoch verbleibt.

Phase III (1990er Jahre): In der dritten Phase schließlich werden die Phänomene 'hohe Staatsverschuldung', 'hohes jährliches Defizit' und 'geringes Wachstum, hohe Arbeitslosigkeit' in einer Weise miteinander verquickt, daß eine Politik des 'Balanced Budget' als eigenständiger Selbstzweck, nicht aber als Endziel einer wachstums- und beschäftigungsoptimierenden Haushaltspolitik begriffen wird (vgl. Domar 1993). ${ }^{3}$ Dabei geht es dann nicht mehr allein um die Forderung nach einer 'soliden' Finanzpolitik, sondern um den Staat und seine Aufgabenbereiche im allgemeinen:

„Die Attacke gegen die Staatsverschuldung symbolisiert nur einen 'Stellvertreterkrieg', denn das eigentliche Angriffsobjekt ist der Interventionsstaat, weil die Markttheoretiker eine Zurückführung des Staatsanteils am realen Sozialprodukt wünschen." (Lübke 1988: 155)

Die Kernbereiche der Bereitstellung öffentlicher Güter stehen zur Diskussion, restriktive Haushaltspolitik ist zur unhinterfragten 'pensée unique' geworden. ${ }^{4}$ Die implizite Rückführung der öffentlichen Ausgaben- und Steuerquote verschwindet in der bundesdeutschen Haushaltsentwicklung hinter der historischen Einmaligkeit der Finanzierungserfordernisse der deutschen Einheit, läßt sich aber in anderen europäischen Ländern gut nachvollziehen.

In der Bundesrepublik kam den ,TINA-Rhetorikern' ein kontinuierlicher Anstieg der öffentlichen Schuldenquote von 20\% in den frühen 1970er Jahren auf über 60\% Ende der 1990er Jahre zwar gerade recht, um Kritiker einer eindimensionalen Sparpolitik als ,Ewiggestrige ${ }^{6} \mathrm{zu}$ brandmarken (vgl. z.B. Hüther/Wolff 1994), doch zeigt z.B. die Entwicklung in Großbritannien, dass es selbst bei kontinuierlich fallender Schuldenquote und erfolgreicher Konso-

2 Dieses Interaktionsmuster kann zumindest gezeigt werden, wenn - wie in der Bundesrepublik - der Geldpolitik der autonomen Bundesbank eine 'Stackelberg-Führerschaft' zugestanden wird (vgl. Heise 2000b: 6ff). In Österreich beispielsweise, wo die Geldpolitik fast vollständig an die Vorgaben der Bundesbank gekoppelt ist und deshalb eine Interaktion mit den nationalen Politikträgern nur in Maßen erfolgt, konnte die Finanzpolitik einen expansiveren Kurs fahren.

3 Einer der renommiertesten, schulenungebundenen Ökonomen der Gegenwart - Willem Buiter - verweist auf die ökonomische Unsinnigkeit einer Politik des 'Balanced Budget': „Both the 'classical' and the 'keynesian' approaches to financial policy reviewed in this paper force one to conclude that a balanced budget policy is very likely to be harmful in a wide range of circumstances." (Buiter 1983: 68).

4 Unter welchen sozio-ökonomischen Umständen dieser 'pensée unique' in der Bundesrepublik entstehen konnte, habe ich in Heise (1999) und Heise (2000a: 449ff.) beschrieben. 
liderung durch finanzpolitische Expansion gleichwohl gelingen kann, nicht nur ein Klima des Staats- und Interventionsskeptizismus zu schaffen, sondern sogar der eigenen Politik die Konnotation der Sparsamkeit zu geben.

\section{Die Politik der öffentlichen Haushalte - in Zahlen gegossene politische Gestaltung statt wirtschaftspolitischer Administration}

Karl Mannheim (1936) hat darauf verwiesen, daß Politik ohne Optionen keine 'Politik' sondern lediglich 'Administration' ist. Wer also bestreitet, dass es unterschiedliche Konzeptionen von Wirtschaftspolitik im allgemeinen und Finanzpolitik im besonderen gibt - Gerhard Schröder prägte die Sentenz von der 'modernen' Wirtschaftspolitik als Ersatz für sozialdemokratische oder konservative Wirtschaftspolitik -, der ist entweder von der genannten 'pensée unique' gänzlich geblendet oder glaubt, ein zeitgeistiges Makro-Klima ${ }^{5}$ für seine politischen Zwecke nutzen zu können. Bevor wir diese genuin politökonomische Frage etwas weiter beleuchten wollen, müssen wir uns zunächst weiter mit der 'Policy-Ebene' - also dem materiellen Teil der Lehre der Wirtschaftspolitik - befassen: In einer modellgestützen Wissenschaft wie der Ökonomie darf es nicht verwundern, daß die Wissenschaftler die reale Welt immer durch den Filter ihrer Modelle sehen. $\mathrm{Ob}$ man an einer gleichgewichtszentrierten Weltsicht à la Léon Walras festhält oder eine Betrachtung bevorzugt, die Raum für Krisen, fehlerhafte Entwicklungen und folglich Interventionen hat, unterliegt im wesentlichen einer ideologischen Prädisposition des Wissenschaftlers - wobei 'Ideologie' hierbei respektive mit 'positives Idealbild' wie 'Verschleierung tatsächlicher Begebenheiten' übersetzt werden kann. Auch der empirische Test - der Blick in die reale Welt - ermöglicht in einer nicht-experimentellen Disziplin nicht wirklich die Diskriminierung zwischen den Modellen.

Es bleibt die Überzeugungskraft der Grundannahmen, ohne die keine Theorie auskommt. Immerhin hält ein - wenngleich immer kleinerer - Kreis von Fachökonomen die Annahme tauschtheoretischer Grundstrukturen moderner Gesellschaften und die Rationalitätsannahme walrasianischer Theorien für unzulänglich - ohne diese aber ließe sich die finanzpolitische Skepsis des Barro-Ricardo-Äquivalenztheorems (BRÄT) kaum ernsthaft begründen ${ }^{6}$ und die folgende Beschreibung der Fragestellung der walrasianischen Finanztheo-

5 Mit Makro-Klima sind herrschende gesellschaftliche Leitbilder wie Individualität oder Kollektivität, Egoismus oder Solidarität, Materialismus oder Post-Materialismus etc. gemeint; vgl. Heise (2000b: 450).

6 Wenn hier das BRÄT des Rationalen-Erwartungs-Monetarismus als Leitbild der walrasianischen Finanzpolitik ausgewählt wird, dann nicht etwa um einen leicht zu attackierenden Strohmann zu konstruieren, sondern ausschließlich, weil das BRÄT die konsequenteste Formulierung der Logik der Politikunwirksamkeit ist und deshalb einer finanzpolitischen Konzeption die Grundlage liefern kann. Vgl. hierzu Manfred Streit (2000: 298). 
rie bliebe unverständlich: „Nicht die Stabilisierungsleistungen der Fiskalpolitik in einer instabilen Wirtschaft werden untersucht, sondern die Destabilisierungen eines stabilen Systems qua Fiskalpolitik“" (Duwendag 1980: 59).

Die (neo-)walrasianische Theorie geht davon aus, daß die Finanzpolitik nicht in der Lage sei, einen dauerhaften Einfluss auf das Wirtschaftswachstum auszuüben - wenn aber das Wirtschaftswachstum quasi exogen gegeben ist (,natürliche Wachstumsrate), dann bleibt es zunächst unbestimmt, ob der staatliche Akteur sich verschulden (finanzpolitische Expansion) oder aber sparen (finanzpolitische Restriktion) soll. Vor dem Hintergrund einer Vollbeschäftigungssituation - allerdings auch nur dann - bedeutet öffentliche Verschuldung entsprechende private Sparsamkeit - ein Phänomen, das besser bekannt ist als ,crowding out' (Verdrängung) privater Ausgaben (Konsum- und Investitionsausgaben) durch öffentliche Verschwendung. Wenn dann unterstellt wird - was gewöhnlich geschieht -, dass private Mittelverwendungen per se, d.h. ohne weitere Erklärungsnotwendigkeit, effizienter sind als öffentliche Ausgaben, wird die Rationalität ausgeglichener und möglichst beschränkter öffentlicher Haushalte deutlich. Empirisch kaum ernsthaft bestreitbare Effekte der Finanzpolitik werden dann entweder auf kurzfristige Ungleichgewichtssituationen - also konjunkturelle Phänomene - oder auf die beschränkte Rationalität der Wirtschaftssubjekte geschoben.

$\mathrm{Ob}$ der Siegeszug dieser walrasianischen Modellwelt in der ökonomischen Disziplin auf die hohe Reproduktionsfähigkeit der beteiligten Wissenschaftlern oder die formale Eleganz des Modells zurückzuführen ist, sei dahingestellt, er ist gewiss nicht durch den überlegenen empirischen Gehalt - also den offensichtlichen Einklang mit der realen Welt - gedeckt. Die Akzeptanz der aus dieser Modellwelt ableitbaren, restriktiven Haushaltspolitik auf der Politics-Ebene - also unter Einbeziehung der Interessengebundenheit von Wirtschaftspolitik - scheint einfacher zu erklären zu sein: Einerseits dient sie den Interessen der Meritokratie (vgl. Heise 1999), die bei zunehmender Homogenität des sozioökonomischen und soziokulturellen gesellschaftlichen Kontextes insbesondere der politischen Einflußträger stärker als früher gesellschaftliche Weltbilder prägen. Andererseits darf nicht unterschätzt werden, welche Bedeutung unter den Bedingungen unsicherer Anschlußkommunikation vom wirtschaftlichen zum politischen System simplen, eingängigen Symbolen im (wirtschafts-)politischen Prozess zukommen kann. Das 'Balanced Budget', der 'Sparhaushalt' mag ein solches Symbol sein, dessen intuitive Eingängigkeit auf einen Selbsterfahrungswert der Individuen (die in diesem Zusammenhang vor allem Wähler sind) setzen kann. Schließlich kann es in einer Welt, in der ökonomische Zusammenhänge sehr komplex sind und nur bedingter politischer Steuerbarkeit unterliegen, sinnvoll sein, den Instrumenteneinsatz und nicht die Erreichung von Zielen zum Erfolgskriterium der eigenen Politik zu stilisieren. 
Für mich steht aber außer Frage, daß die Popularität des walrasianischen Modells auch mit den Problemen der standard- und linkskeynesianischen Alternativen zu tun hat. ${ }^{7}$ Auf der theoretischen Ebene ist es den mitunter prominenten Standard- und Linkskeynesianern nicht gelungen, die für das Gleichgewichtsmodell zentrale Annahme der Gültigkeit des Walrasschen Gesetzes bzw. dessen klassischen Pendants - des Sayschen Theorems - konsistent zu widerlegen. ${ }^{8}$ Dieses Versäumnis implizierte - bei aller berechtigten Kritik an manch unrealistischer Annahme im 'protective belt' des walrasianischen Paradigmas - die Möglichkeit der Opponenten, die Alternativen zu bloßen 'Theorien der Depression' (also kurzfristigen Abweichungen vom Pfad der Glückseligkeit) zu stempeln und verleitet die Befürworter zu einem cartesianischen Interventionsoptimismus, der gewissermaßen das Pendant zum Selbststeuerungsoptimismus der walrasianischen Theorie abgibt. Für die hier zur Diskussion stehende Finanzpolitik aber ist die Zyklik des Krisenphänomens von besonderer Bedeutung, denn sie ermöglicht es einerseits, die Frage nach der Nachhaltigkeit einer gegensteuernden Finanzpolitik auszublenden und reduziert andererseits die Komplexität der Ursachen- und Wirkungsanalyse enorm. Die wirtschaftspolitische Trinität der Alternativen - Lohnsteigerungen und Umverteilung zur Förderung der Massenkaufkraft, staatliches Defizit-spending zur Abschöpfung brachliegender privater Ersparnisse und geldpolitische Expansion - mag theoretisch unzureichend sein', sicher aber ist der Neuigkeitsgehalt gegenüber den diskreditierten Nachfragepolitiken der 1970er Jahre zu gering, um in 'modernen Zeiten' über den engen Kreis der Adepten hinaus Anhänger zu finden.

Die heterodoxe Forschung der letzten zwei bis drei Jahrzehnte hat auf der theoretischen Ebene mit der Entwicklung eines geldwirtschaftlichen Paradigmas (oder, in Keynes'scher Terminologie, einer monetären Theorie der Produktion) die Grundlage für das Verständnis einer dauerhaft, eben nicht nur zyklisch vom Vollbeschäftigungsgleichgewicht abweichenden kapitalistischen Ökonomie gelegt ${ }^{10}$, in dem ein solcher Zustand nicht als 'Krise', als Marktversagen verstanden werden muß, sondern als Normalzustand einer durch Rationalkalküle unter Unsicherheitsbedingungen (in einer nicht-ergodischen

7 Unter ,Standardkeynesianismus ${ }^{6}$ ist die Lehrbuchdarstellung der Keynesianischen Makroökonomik gemeint, wie sie von Sir John Hicks (1937) begründet wurde und in Deutschland insbesondere von Karl Schiller in Wissenschaft und Politik vertreten wurde, mit ,Linkskeynesianismus' sind jene Versuche gemeint, die die marxistische Unterkonsumtionstheorie mit der standardkeynesianischen Theorie mangelnder effektiver Nachfrage zu verbinden suchten und in Deutschland insbesondere von der ,Memorandumgruppe vertreten wird.

8 Vgl. hierzu die Kontroverse um die Ungleichgewichtstheoretiker der ,Neuen Keynesianischen Makroökonomik': u.a. Clower (1965); Leijonhufvud (1968); Malinvaud (1977). Kritisch dazu: Gale (1981); Priewe (1984), zusammenfassend: Hagemann/Kurz/Schäfer (1981).

9 Man vergleiche dazu z.B. die Kontroverse zwischen Riese (1979) und Hickel (1979).

10 Vgl. u.a. Davidson (1978); Davidson (1994); Palley (1996); Heise (1991); Betz (1993). 
Welt) gesteuerten Volkwirtschaft. Der durch die Interdependenzen der Handlungen der Akteure erkennbare Komplexitätsgrad muß auf der 'Policy-Ebene' zwar nicht zum wirtschaftspolitischen Nihilismus eines Non-Dezisionismus (vgl. Riese 1998), wohl aber zur Anerkennung einer lediglich bedingten Steuerbarkeit führen. Vor allem aber stellt sich für die Finanzpolitik des staatlichen Akteurs nun ganz offensichtlich die Frage der Nachhaltigkeit: Wie kann dauerhafte Intervention und/oder sozialpolitische Abfederung finanziert werden, ohne die Grenzen der finanziellen Belastbarkeit des Staates zu überschreiten? Gibt es solche Grenzen überhaupt und wie lassen sie sich bestimmen?

\section{Finanzpolitik zwischen Notwendigkeit und Machbarkeit - Nach- haltigkeit als ein Konzept überzyklischer Interventionspolitik}

Auf der Grundlage eines postkeynesianischen Modells (vgl. z.B. Heise 1991; Betz 1993) läßt sich mit der Unterscheidung eines systemischen und eines institutionellen Defekts die dauerhafte Interventionsnotwendigkeit der öffentlichen Haushaltspolitik in reifen marktwirtschaftlich organisierten Ökonomien ebenso begründen wie die Notwendigkeit zur Verhaltensabstimmung der sozio-ökonomischen Politikträger - zu letzterem später noch mehr. Zunächst geht es um die Erstellung öffentlicher Güter durch den staatlichen Akteur. Dabei kann der Bedarf an öffentlicher Güterversorgung mittels der klassischen 'Theorie des Marktversagens' (Allokationsfunktion) ebenso ermittelt werden wie durch normative Zielsetzungen (Distributions- und Stabilisierungsfunktion) mittels politischem Diskurs. Soziale Sicherung wie auch Vollbeschäftigung zumindest auf dem Niveau entwickelter Wohlfahrtsstaaten müssen Gegenstand der politischen Willensbildung sein und können nicht mehr allein aus den Funktionsbedingungen des kapitalistischen Systems heraus abgeleitet werden. ${ }^{11}$ Eine Unterscheidung in konsumtiv und investiv verwendete öffentliche Güter erscheint von einem analytischen Standpunkt aus zweierlei Gründen gerechtfertigt: (1) Im Hinblick auf die Ressourcenaufbringung muß differenziert werden, ob die Erstellung der öffentlichen Güter steuer- oder - zumindest anteilig - kreditfinanziert werden soll; (2) im Hinblick auf die Interventionsursache sollte stärker als im Standard- und Linkskeynesianismus differenziert werden zwischen den verschiedenen Komponenten der gesamtwirtschaftlichen Nachfrage: Verantwortlich für 'the longterm problem of full employment' ist eine systembedingte Investitionsschwä-

11 Während in der traditionellen Theorie Arbeitslosigkeit als 'Marktfehler' verstanden würde und Vollbeschäftigung dann als Funktionsbedingung gelten könnte, so gilt dies im Verständnis der postkeynesianischen Theorie mit ihren multiplen Beschäftigungsgleichgewichten offensichtlich nicht. Vollbeschäftigung ist hier eine spezielle Situation, deren Herbeiführung durch politische Willensbekundung und entsprechende Beschäftigungspolitik erst erzeugt werden muß. 
che, die nicht eigentlich auf einer Unterkonsumtion, sondern einer liquiditätspräferenztheoretischen Determinantion der Opportunitätskosten der Investition - des Zinssatzes - beruht. Mit dem späten Keynes (vgl. Keynes 1943/1980) können wir also zwischen einem 'laufenden Budget' ('current budget') und einem 'Investitionsbudget' ('capital budget') unterscheiden, wobei im laufenden Budget alle nicht-investiven Ausgaben wie Sach- und Personalaufwand, Sozialtransfers etc. erfaßt werden, während im Investitionsbudget lediglich die investiven Ausgaben verbucht werden. Das laufende Budget muss seiner Natur nach im zyklischen Durchschnitt ausgeglichen werden durch entsprechende Steuereinnahmen, seine Höhe richtet sich nach dem politisch vermittelten Wunsch auf Inanspruchnahme öffentlicher Güter mit wesentlich allokativer und distributiver Funktion. ${ }^{12}$ Das Investitionsbudget hingegen übernimmt die Stabilisierungsfunktion in jenem Ausmaß, wie der politische Wille eine Annäherung an Vollbeschäftigung erwünscht. ${ }^{13}$ Mit Hilfe des Investitionsbudgets versucht der Staat also die Volkswirtschaft in der Nähe des Potentialpfades zu halten - hier nun kommt die Notwendigkeit einer Kreditfinanzierung öffentlicher Güterbereitstellung ins Spiel und die Frage nach deren Grenzen. Die Höhe des Investitionsbudgets hängt von der gewünschten Infrastrukturausstattung und der 'Investitionslücke' - also der Differenz zwischen 'Vollbeschäftigungsersparnis' und privater Investitionstätigkeit - ab, der Anteil der dauerhaften Kreditfinanzierung muß sich dem Nachhaltigkeitsanspruch stellen.

Mit Luigi Pasinetti (1998) und Olivier Blanchard et al. (1990) können wir 'Nachhaltigkeit' (sustainability) als jenes Haushaltsdefizit (oder -überschuß) definieren, das eine als optimal empfundene Schuldenquote zumindest stabilisiert. Unter gewissen Bedingungen ${ }^{14}$ bedeutet dies gleichzeitig die Stabilisierung eines Primärbudgets - also die Konstanz der finanzpolitischen Handlungsspielräume. Es läßt sich leicht zeigen, daß die in diesem Sinne 'nachhaltige' Defizitquote $(\mathrm{S} / \mathrm{Y})$ von der Wachstumsrate des BIP (g) und der 'optimalen' Schuldenquote $(\mathrm{D} / \mathrm{Y})$ bestimmt wird:

12 Wobei die soziale Abfederung mit distributiver Ausrichtung natürlich auch stabilisierende Funktion übernehmen kann und soll.

13 Selbstverständlich werden öffentliche Investitionen in jenen Feldern getätigt, die - aufgrund von Marktversagen oder kollektiver Bestimmung (meritorische Güter) - privaten Investitionen verschlossen bleiben. In diesem Sinne übernimmt das Investitionsbudget auch Allokationsfunktionen.

14 Die Bedingung - unrealistisch wohl, aber praktisch von nachrangiger Bedeutung - ist ein konstanter Zinssatz auf die öffentliche Schuld. Die Annahme wird nur dann wirklich problematisch, wenn wir eine Inflationierung zum Abbau der Realschuld (Inflationssteuer) zuließen - dann nämlich müßte sich dies in einer Erhöhung des Zinssatzes und folglich einer Beschränkung der finanzpolitischen Spielräume niederschlagen. 


$$
\mathrm{S} / \mathrm{Y}=\mathrm{g}(\mathrm{D} / \mathrm{Y})
$$

mit $S$ = öffentliche Netto-Neuverschuldung; $Y=$ Volkseinkommen; $\mathrm{g}=$ Wachstumsrate des Volkseinkommes; $\mathrm{D}=$ öffentlicher Schuldenstand

Für den Primärhaushalt ergibt sich:

$$
\mathrm{S}_{\mathrm{P}} / \mathrm{Y}=(\mathrm{i}-\mathrm{g})(\mathrm{D} / \mathrm{Y})
$$

mit $S_{P}=$ Primärüberschuss; $i=$ Zinssatz auf öffentliche Schuldpapiere

Es ist leicht einsichtig, daß eine hohe 'optimale' Schuldenquote leichter zu stabilisieren ist (also eine höhere 'nachhaltige' Defizitquote erlaubt) als eine niedrigere, andererseits eine niedrigere 'optimale' Schuldenquote einen größeren finanzpolitischen Handlungsspielraum zuläßt als eine höhere 'optimale' Schuldenquote. Es ergibt sich also ein trade off zwischen heutiger Neuverschuldungsmöglichkeit (mit den entsprechenden Vorteilen für den staatlichen Akteur) und der künftigen Handlungsbeschränkung (mit den entsprechenden Nachteilen für den staatlichen Akteur; vgl. Abb. 1): Wir wollen annehmen, der staatliche Akteur - die Regierung - bilde sich eine Vorstellung über die Abwägung (Präferenzen) von gegenwärtigem Nutzen zusätzlicher Handlungsmöglichkeiten durch Neuverschuldung einerseits und künftiger Handlungsbeschränkung bei steigendem, notwendigen Primärüberschuß andererseits ${ }^{15}$ - damit läßt sich nun eine 'optimale' Schuldenquote (bei gegebenem Wirtschaftswachstum $\mathrm{g}_{1}$ ) bestimmen. Das Optimalitätspostulat impliziert dabei die Nachhaltigkeit der Finanzpolitik, wie auch die intertemporale Abwägung des staatlichen Akteurs. ${ }^{16}$

Klar wird nun auch, daß die Netto-Neuverschuldung, die mit der 'optimalen Schuldenquote' korreliert, nicht unabhängig von der Wachstumsrate $\mathrm{g}$ bestimmt werden kann, ja daß sich selbst die 'optimale Schuldenquote' mit der Wachstumsrate verändern kann. Anders gesagt: die Vorgabe einer maximalen Netto-Neuverschuldungsgrenze und einer 'optimalen' Verschuldungsquote in den Protokollen zum Maastrichter Vertrag wäre nur dann sinnvoll, wenn

15 Die Abwägung wird dargestellt durch die Indifferenzkurven - eine theoretischen Konstruktion, die die intertemporale Substitution zweier Zustände (hier: Netto-Neuverschuldung und Primärüberschuss) bei unverändertem Nutzenniveau darstellt -, wobei die flachere Indifferenzkurve I1 gebenüber I2 eine höhere Wertschätzung der gegenwärtigen gegenüber den zukünftigen Gestaltungsmöglichkeiten impliziert. Im Tangentialpunkt der Indifferenzkurve mit der Nachhaltigkeitslinie als ,Budgetbeschränkung' ergibt sich dann die optimale intertemporale Allokation zwischen heutigen und künftigen Handlungsmöglichkeiten unter Sicherung der Nachhaltigkeit.

16 Je kürzer der Amtszeitraum einer Regierung und je geringer die Wiederwahlchancen, desto höher dürfte die Präferenz für heutige gegenüber künftigen Handlungsmöglichkeiten sein vielleicht auch ein Grund, weshalb ein Land wie Italien eine deutlich höhere Staatsschuldenquote aufweist als etwa die Bundesrepublik. 
Abbildung 1: Optimale Verschuldung

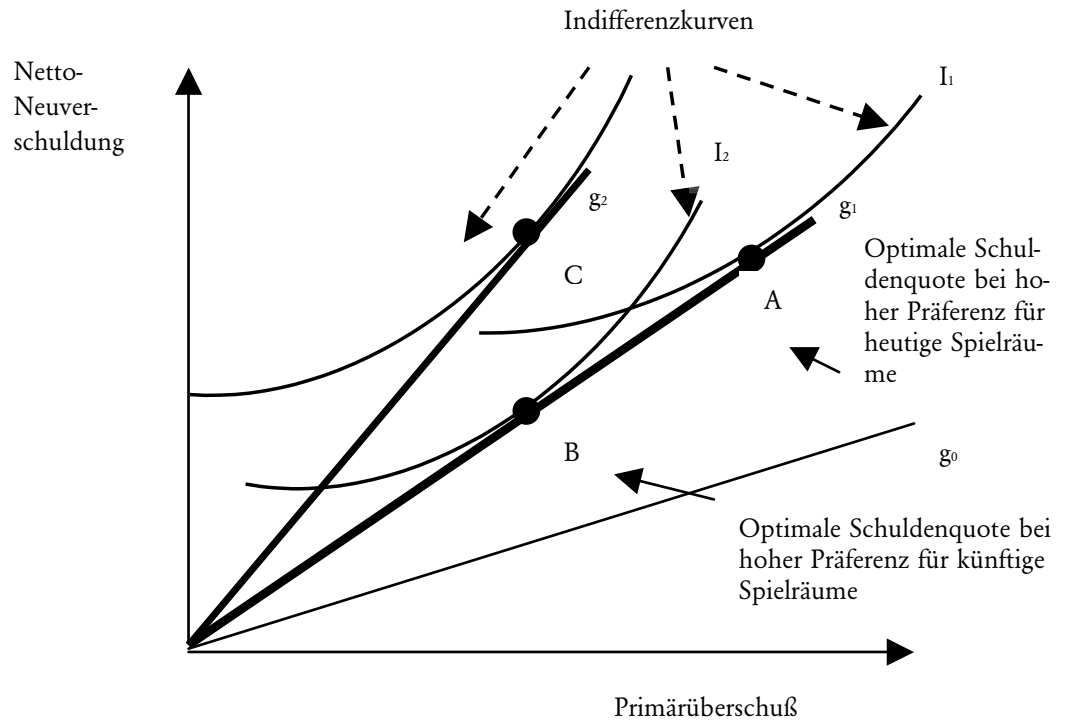

Anmerkung: Die Nachhaltigkeitslinien sind in Abhängigkeit verschiedener Wachstumsraten $\mathrm{g}_{\mathrm{i}}$ dargestellt mit $g_{0}<g_{1}<g_{2}$. Entlang der Nachhaltigkeitslinie steigt die stabile Schuldenquote an, d.h. in Punkt A wird bei höherer Netto-Neuverschuldung und höherem Primärüberschuß eine höhere Schuldenquote stabilisiert als in Punkt B.

ausgeschlossen werden könnte, daß der Kurs der Finanzpolitik selbst einen dauerhaften Einfluß (im Gegensatz zu einem temporären Einfluß im Sinne eines Anschub- oder Strohfeuereffektes) auf die Wachstumsrate $g$ hat. Dies ist natürlich die Position der gegenwärtig dominanten walrasianischen Theorie, entspricht aber weder den empirischen Fakten der bundesdeutschen Entwicklung ${ }^{17}$ noch den Prognosen der postkeynesianischen Theorie.

Allerdings geben weder die postkeynesianische Theorie noch die hier dargelegten Ausführungen einen Freibrief für eine expansive Finanzpolitik jedweder Art - öffentliche, kreditfinanzierte Ausgaben sind nur dann sinnvoll und zulässig zur Erfüllung der Stabilisierungsfunktion, wenn sie die Nachhaltigkeit der Finanzpolitik nicht gefährden. Dies bedeutet, dass sie einen Prozess der Einkommenserzeugung auslösen müssen (also eine Verschiebung der Nachhaltigkeitskurve in Abb. 1 z.B. von $g_{1}$ auf $g_{2}$ ), der die Stabilisierung der als 'optimal' bestimmten Schuldenquote ermöglicht. Der Einkommensmulti-

17 In einer jüngsten Veröffentlichung weist Lombard (2000: 329) den langfristigen Einfluß der (restriktiven) Finanzpolitik in der Europäischen Union nach. 
plikator einer nachhaltigen Finanzpolitik ergibt sich aus dem reziproken Wert der 'optimalen' Schuldenquote (vgl. Heise 1996). Bei einer laut Maastrichter Vertrag als 'optimal' empfundenen Schuldenquote von 0,6 (also 60\%) in der $\mathrm{E}(\mathrm{W}) \mathrm{U}$ ergibt sich ein benötigter Einkommensmultiplikator von 1,67. ${ }^{18}$ Damit ergibt sich ein weiteres Kriterium, nach dem staatliche Ausgaben als 'investiv' qualifiziert werden können: Jene Ausgaben, deren Einkommensmultiplikator groß genug ist, um eine 'optimale' Schuldenquote zu stabilisieren - in diesem Sinne also gesellschaftlich 'rentabel' sind - werden als investiv bezeichnet und müssen folglich nicht aus dem Steueraufkommen finanziert werden. In ökonometrischen Modellen werden Multiplikatoren bei investiver Verwendung von etwa 2,0 berechnet (vgl. Heise 1996: 709), Scherf (1985: 360f.) beispielsweise berechnet für den herrschenden Ausgabenmix in der Bundesrepublik Anfang der 1980er Jahre einen Multiplikator von 1,75. ${ }^{19}$ Wenn Vollbeschäftigung als wirtschaftspolitisches Ziel normativ festgelegt wurde - und zumindest auf der rhetorischen Ebene halten alle Parteien in Deutschland an diesem Ziel fest -, dann muß allerdings eine 'expansive' - d.h. investionsorientierte und kreditfinanzierte - öffentliche Haushaltspolitik solange betrieben werden, wie die zusätzlichen Ausgaben einen hinreichend hohen Multiplikator erwarten lassen und weiterhin unausgelastete Ressourcen - also Arbeitslosigkeit - bestehen. Jeder Verzicht auf eine entsprechende finanzpolitische Expansion muß als volkswirtschaftlich suboptimal verstanden werden. ${ }^{20}$

\section{Sicherung der Nachhaltigkeit durch Kooperation - Design einer 'integrativen Wirtschaftspolitik'}

Bisher klingt die Politik nachhaltiger öffentlicher Haushalte reichlich 'hydraulisch' - dies muß nun in Frage gestellt werden. Wie immer in der simplen Modellwelt, so habe auch ich an mancher Stelle implizite Annahmen getroffen, die nicht recht realitätsnah sind: Einerseits habe ich mögliche Auswirkungen einer 'expansiven' Finanzpolitik auf das Preisniveau noch nicht berücksichtigt, andererseits einen konstanten Zinssatz - also keine geldpoliti-

18 Dies ist der Multiplikator des öffentliches Defizits, also der um die erhöhten Steuereinnahmen bereinigten zusätzlichen öffentlichen Ausgaben. Der (geringere Brutto-)Multiplikator dieser zugrundeliegenden öffentlichen Ausgaben errechnet sich aus (1-t)/ $k$, wobei $t$ die Steuerquote angibt. Bei realistischen Werten für $\mathrm{t}=0,25$ und $\mathrm{k}=0,6$ ergibt sich ein Ausgabenmultiplikator von 1,25 .

19 Die damals zu stabilisierende Staatsschuldenquote von unter $40 \%$ hätte einen Defizitmultiplikator von 2,5 und einen Ausgabenmultiplikator von etwa 1,9 erfordert, weshalb dieses Unterfangen beim herrschenden Ausgabenmix nicht gelingen konnte.

20 Erstaunlicherweise werden in der neueren Literatur unter Staatsverschuldung nur die Verschuldungsgrenzen thematisiert, nicht aber die Verschuldungsspielräume, die es zu nutzen gilt. Eine rühmliche Ausnahme ist Tiepelmann/Dick (1995: 151). Dies war einmal anders, als unter der Bezeichnung 'kompensatorische Finanzpolitik' ein dem hier vorgestellten Ansatz ähnliches Konzept entwickelt wurde (vgl. z.B. Böttger/Gretschmann/Huppertz 1981), das allerdings den Nachhaltigkeitsaspekt weitgehend vernachlässigte. 
schen Reaktionen und Präferenzveränderungen der Finanzmarktakteure - unterstellt. Es dürfte einer der Kardinalfehler des hydraulischen Keynesianismus gewesen sein, einerseits die Diskriminierungsgrenzen zwischen Preis- und Mengeneffekten der Finanzpolitik zu wenig beachtet, andererseits die Konfliktpotentiale unkoordinierter wirtschaftspolitischer Akteure unterschätzt zu haben. Beides zusammen ergibt den institutionellen Defekt hochentwickelter kapitalistischer Volkswirtschaften: Bei unbestreitbaren Interdependenzen der verschiedenen Politikbereiche (vgl. Nordhaus 1994; Heise 2000b) - Finanz-, Geld- und Lohnpolitik sind gemeint - kommt es ohne institutionelle Vorkehrungen zur gegenseitigen wachstums- und beschäftigungshemmenden Blockade des sogenannten 'Gefangenen-Dilemmas'. Insbesondere die Geldpolitik ist restriktiver als es für den Erhalt der Preisstabilität allein notwendig wäre, die Finanzpolitik muß sich - zumindest bei vollständiger Notenbankautonomie - in eine 'hierarchische Koordination' begeben, die bei hoher 'passiver' Neuverschuldung (vgl. Heise 1996; Nowotny 1999: 605ff.) immer weniger Handlungsspielraum läßt. Nur eine institutionalisierte Verhaltensabstimmung - ein Makro-Dialog, der mit dem Konstrukt der Konzertierten Aktion des altkeynesianischen Stabilitäts- und Wachstumsgesetzes schon von der Spielcharakteristik wenig gemein hat - kann unter etlichen Nebenbedingungen ${ }^{21}$ das Zuschnappen der 'Kooperationsfalle' verhindern und den „ursprünglich eindimensionale(n) Staatsinterventionismus zu einem umfassenden multivariablen Krisenmanagement entwickeln" (Spahn 1981: 277). Hier besteht institutioneller Reformbedarf, der eine integrative bzw. kooperative Wirtschaftspolitik als Ergänzung zur 'Bündnis-für-Arbeit-Modernisierung' entwirft. ${ }^{22}$ Zugegebenermassen sind trotz rot-grüner Bundesregierung die polit-ökonomischen Rahmenbedingungen gegenwärtig eher ungünstig für eine solche 'integrative Wirtschaftspolitik', da es keinen durchschlagskräftigen 'policy maker' bzw. eine ,advocacy coalition' (vgl. Schmid 2000: 5f.) und schon gar keinen 'führenden Kopf' gibt, der in einer medial geprägten Welt ein Gegengewicht zur herrschenden 'pensée unique' abgeben könnte.

\section{Zusammenfassung und Ausblick}

Es wäre zu wünschen, daß die Finanzpolitik wenigstens wieder stärker Gegenstand einer offenen, kontroversen Diskussion in Wissenschaft und Politik würde - wenn es also gelänge, das Meinungskartell der 'pensée unique' zu

21 Nebenbedingungen und Institutionen sind ausführlicher beschrieben in Heise (1999); Hein/Heise (1999); Heise (2000b). Hierin wird auch der skeptischen Perspektive einer Verhaltensabstimmung in Fritz W. Scharpfs bahnbrechendem Werk ,Sozialdemokratische Krisenpolitik' (1987) unter veränderten Rahmenbedingen widersprochen.

$22 \mathrm{Zu}$ den Mustern der Reform- bzw. Modernisierungsdebatte vgl. u.a. Schmid/Niketta (1998); Heinze/Schmid/Strünck (1999); Heinze (1998); Arlt/Nehls (1999); Urban (2000). 
brechen. ${ }^{23}$ Dann könnte frei über die gesellschaftlich gewünschte, 'optimale' Staatsverschuldung bzw. deren Veränderung angesichts einer zunehmend auf Generationengerechtigkeit pochenden jüngeren Generation diskutiert werden - was im Übergang von einem Gleichgewicht zum anderen (vgl. Abb. 1) tatsächlich mit einem temporär ausgeglichenen oder gar überschüssigen Budget verbunden sein $\mathrm{kann}^{24}$. Aber gleichzeitig wären auch die (negativen) Wachstums- und Beschäftigungseffekte, die dies für den Übergang bedeuten kann, $\mathrm{zu}$ thematisieren. ${ }^{25}$ Unabhängig davon muß der Mythos vom 'Balanced Budget', der in den 1970er Jahren schon einmal auf dem Friedhof der Dogmengeschichte begraben $\operatorname{schien}^{26}$, als Versuch gewertet werden, das politische Symbol (vgl. Savage 1988) einer 'good governance' für eine Beschränkung der Staatstätigkeit und für einen Interventionsskeptizismus, der bar jeder soliden ökonomischen Fundierung ist, zu mißbrauchen. Eine nachhaltige Finanzpolitik jenseits dieser 'Symbolpolitik' muß ihre Gestaltungs- und Finanzierungsgrenzen akzeptieren, gleichzeitig aber auch die vorhandenen Spielräume trotz manchen politischen und ökonomischen Risikos nutzen. Das ökonomische Risiko kann durch einen Makro-Dialog, also eine institutionell abgesicherte Kooperation der sozio-ökonomischen Akteure gemindert, die Ziel-MittelKonformität auch der Finanzpolitik wesentlich gesteigert werden.

Zweifellos aber benötigt der damit eingeforderte Paradigmenwechsel in der Wirtschaftspolitik eine Veränderung des herrschenden, meritokratischen Weltbildes. Es würde den Rahmen dieser Arbeit sprengen, wollte ich den Voraussetzungen eines solchen Wandels des dominanten Weltbildes an dieser Stelle nachgehen (ausführlicher dazu: Heise 2001). Sicher scheint aber, daß es ,modischen Schwankungen' unterliegt, die vielleicht mit dem ,Grad der Frustration', also dem Grad der Abweichungen der Versprechungen des Weltbildes von der sozialen Realität, zusammenhängen (vgl. Screpanti 1999: 37ff.). Was heißt dies konkret? Der keynesianische Interventionsstaat kam unter Druck, als die Verheissung eines wohlgesteuerten sozio-ökonomischen Gleichgewichts (Globalsteuerung) in der Stagflation der frühen 1980er Jahre unter-

23 In Österreich wird gegenwärtig heftig um die Meinungsführerschaft gekämpft; vgl. z.B. BEIGEWUM (2000).

24 Wenn allgemeiner politischer Konsens über die Rückführung der Schuldenquote als höchster finanzpoltischer Priorität bestände, liesse sich die anfänglich benannte Einmütigkeit der Konsolidierungsnotwendigkeit jenseits allem wissenschaftlichen und politischen Dissens erklären - allerdings nur für die Zeit des Übergangs z.B. von Punkt A nach Punkt B in Abbildung 1, nicht als immerwährende Haushaltsregel.

25 Vgl zur Diskussion um die Konsolidierungseffekte z.B. Marterbauer/Walterskirchen (1999).

26 So konnten Böttger/Gretschmann/Huppertz (1981: 151) fröhlich formulieren: „Der Grundsatz eines jährlichen Budgetausgleichs, d.h. das klassisch-orthodoxe Postulat einer weitgehend schuldenfreien Staatswirtschaft, wurde bis in die jüngste Vergangenheit kaum noch erhoben. Zu eindeutig haben die Funktionsanforderungen einer entwickelten kapitalistischen Industriegesellschaft inzwischen die Unhaltbarkeit einer Norm aufgezeigt, nach der die öffentliche Finanzwirtschaft entsprechend den Regeln eines privaten Haushalts geführt werden müßte.“ 
unterging. Bereits vorher begann sich das ,Mikro-Klima“ - die akzeptierten (wirtschafts-)politischen Konzeptionen - durch den Siegeszug des Monetarismus und der daraus ableitbaren Angebotspolitik zu wandeln und neue, vernachlässigte Prioritäten wurden auf der politischen Agenda festgeschrieben: Eigenverantwortlichkeit, unternehmerische Dynamik, Preisstabilität. Hilfreich waren hierbei eine weitgehend homogene soziale Herkunft der Politik-, Wirtschafts- und Medieneliten aus dem höheren Bildungssegment und aus selbständigen Berufen (vgl. Rebenstorf 1997) wie auch der Wandel der meinungsformenden Medienlandschaft von der Dominanz religiöser und politischer Ideologieträger zu derjenigen von Wirtschaftsbetrieben mit klarem unternehmensideologischem Informationsbias. ${ }^{27}$ Die Interpretation der Weltrealität erfolgt deshalb zunehmend aus Unternehmenssicht, nämlich durch eine gesellschaftliche Elite, für die individuelle Leistungsbereitschaft Priorität vor Solidarität und staatlicher Interventionsverpflichtung genießt (vgl. Kaina 1997). Soll sich dies ändern, so bedarf es unter den aktuellen Bedingungen einerseits einer wissenschaftlich legitimierten, in ein ,marketingfähiges' Programm übersetzbaren wirtschaftspolitischen Alternative, andererseits einer zunehmenden Frustration (im obigen Sinne) in bezug auf das vorherrschende neoliberale Weltbild. Zu Ersterem wollte diese Arbeit einen bescheidenen Beitrag leisten, Letzteres dürfte eine Frage der Zeit und der Fähigkeit der Meritokraten sein, erneut Sachzwangargumente zu entwickeln oder Sündenböcke für die Fehlschläge der von ihnen favorisierten Wirtschaftspolitik zu entdecken.

\section{Literatur}

Arestis, P., McCauley, K., Sawyer, M. (2001): An alternative stability pact for the European Union; in: Cambridge Journal of Economics, Vol. 25, No.1, S. 113 - 130

Arlt, H.-J., Nehls, S. (Hrsg.), (1999): Bündnis für Arbeit. Konstruktion, Kritik, Karriere, Opladen

BEIGEWUM (Beirat für gesellschafts-, wirtschafts- und umweltpolitische Alternativen) (2000): Mythos Nulldefizit. Alternativen zum Sparkurs, Wien

Betz, K. (1993): Ein monetärkeynesianisches gesamtwirtschaftliches Gleichgewicht, Marburg

Blanchard, O. et al. (1990): The sustainability of fiscal policy: new answers to an old question; in: OECD Economic Studies No. 15, Paris

Böttger, G., Gretschmann, K., Huppertz, P.-H. (1981): Kompensatorische Finanzpolitik als Vollbeschäftigungsstrategie; in: Wirtschaftsdienst, Nr. 3, S. 151 - 156

Buiter, W. (1983): The Theory of Optimum Deficits and Debts; in: The Federal Reserve Bank of Boston (Hrsg.); The Economics of Large Government Deficits. Conference Series No. 27, Boston, S. $46-69$

Clower, R. (1965): The Keynesian Counter-Revolution: A Theoretical Appraisal; in: Hahn, F.H., Brechling, F.P.R. (Hrsg.); The Theory of Interest Rates, London

Davidson, P. (1978): Money and the Real World, London

- (1993): On Debts and Deficits; in: American Journal of Economics and Sociology, Vol. 2, S. $475-478$

27 Dies ist zweifellos ein Aspekt dessen, was als ,systematisches Glück der Kapitalisten` benannt wurde (vgl. Dowding 1996: 71ff.). 
- (1994): Postkeynesian Macroeconomic Theory, Aldershot

Dowding, K. (1996): Power, Buckingham

Duwendag, D. (1980): Monetäre Grenzen der Staatsverschuldung; in: Ders., Siebert, H. (Hrsg.); Politik und Macht, Stuttgart/New York, S. 56 - 81

Gale, D. (1981): Money: in disequilibrium, Cambridge

Gerrard, B. (1993): Competing Schools of Thought in Macroeconomics - An Ever-Emerging Consensus?; in: Journal of Economic Studies, Vol. 23, S. 53 - 69

Hagemann, H., Kurz, H.-D., Schäfer, W. (Hrsg.) (1981): Die neue Makroökonomik, Frankfurt

Hein, E., Heise, A. (1999): Beschäftigungspolitische Möglichkeiten und Beschränkungen des Bündnisses für Arbeit: der fehlende Makro-Dialog; in: WSI-Mitteilungen, H. 12, S. $825-838$

Heinze, R.G. (1998): Die blockierte Gesellschaft, Wiesbaden/Opladen

Heinze, R.G., Schmid, J., Strünck, Chr. (1999): Vom Wohlfahrtsstaat zum Wettbewerbsstaat, Opladen

Heise, A. (1991): Tauschwirtschaft und Geldökonomie, Frankfurt u.a.

- (1996): Aktive versus passive Staatsverschuldung - etwas einfache Arithmetik; in: WSIMitteilungen, H. 11, S. 706 - 709

- (1999): Globalisierung, Arbeitslosigkeit und Ungleichheit - ein polit-ökonomischer Erklärungsversuch; in: v. Bülow, W. et al. (Hrsg.); Globalisierung und Wirtschaftspolitik, Marburg, S. 205 - 232

- (2000a): 'New Politics' - Einige makroökonomische Überlegungen zum Bündnis für Arbeit; in: WSI-Mitteilungen, H. 7, S. $444-453$

- (2000b ): Theoretische Grundlagen einer Verhaltensabstimmung der makroökonomischen Politikträger, Schriftenreihe des Promotionsschwerpunkts Makroökonomische Diagnosen und Therapien der Arbeitslosigkeit, Stuttgart

- (2001):New Politics - Integrative Wirtschaftspolitik für das 21. Jahrhundert, Münster

Hicks, J.R. (1937): Mr. Keynes and the Classics; in: Econometrica, Vol. 5, S. 147 - 159

Keynes, J.M. (1980): The long-term problem of full emplyoment (1943); in: Moggridge, D. (Hrsg.); The Collected Writings of John Maynard Keynes, Vol. 27, London, S. $320 \mathrm{ff}$.

Kaina, V. (1997): Wertorientierungen im Eliten-Bevölkerungsvergleich: Vertikale Distanzen, geteilte Loyalitäten und das Erbe der Trennung; in: Bürklin, W. u.a.; Eliten in Deutschland. Rekrutierung und Integration, Opladen, S. 351 - 389

Leijonhufvud, A.( 1968): On Keynesian Economics and the Economics of Keynes, Oxford

Lindlar, L. (1997): Das mißverstandene Wirtschaftswunder, Tübingen

Lombard, M. (2000): Restrictive Macroeconomic Policies and Unemployment in the European Union; in: Review of Political Economy, Vol. 12, No.3, S. 317 - 332

Lübke, G. (1988): Die Staatsverschuldung im Kontext alternativer Wirtschaftspolitik, Frankfurt u.a.

Malinvaud, E. (1984): The Theory of Unemployment Reconsidered, Oxford

Mannheim, K. (1936): Ideology and Utopia. An Introduction to the Sociology of Knowledge, London

Marterbauer, M., Walterskirchen, E. (1998): Keynesianische oder neoklassische Effekte der Budgetkonsolidierungspolitik in Europa zwischen 1995 und 1997; in: Heise, A. (Hrsg.); Makropolitik zwischen Nationalstaat und Europäischer Union, Marburg, S. 59 - 78

Matzner, E. (2000): Monopolare Weltordnung. Zur Sozioökonomie der US-Dominanz, Marburg

Nordhaus, W.D. (1994): Policy Games: Coordination and Independence in Monetary and Fical Policies; in: Brookings Papers on Economic Activity, No.2, S. 139 - 216

Nowotny, E. (1999): Der öffentliche Sektor, Berlin u.a. (4. Aufl.)

Palley, Th. (1996): Post Keynesian Economics, Basingstoke/London

Pasinetti, L.L. (1998 ): The myth (or folly) of the 3\% deficit/GDP Maastricht 'parameter'; in: Cambridge Journal of Economics, Vol. 22, S. 103 - 116

Priewe, J. (1984): Zur Kritik konkurrierender Arbeitsmarkt- und Beschäftigungstheorien und ihrer politischen Implikationen, Frankfurt

Rebenstorf, H. (1997): Integration und Segmentation der Führungsschicht - Stratifikationstheoretische Determinanten; in: Bürklin, W. u.a.; Eliten in Deutschland. Rekrutierung und Integration, Opladen, S. 123 - 199

Riese, H. (1998): Zur Reformulierung der Theorie der Makropolitik; in: Heise, A. (Hrsg.); Renaissance der Makroökonomik, Marburg, S. 25 - 40

Savage, J.D. (1988): Balanced Budgets and American Politics, Ithaca/London 
Screpanti, E. (1999): Discontinous Changes in Institutional Systems; in: Groenewegen, J., Vromen, J. (Hrsg.); Institutions and the Evolution of Capitalism, Aldershot, S. 30 - 51

Scharpf, F.W. (1987): Sozialdemokratische Krisenpolitik, Frankfurt

Scherf, W. (1985): Budgetmultiplikatoren; in: Jahrbücher für Nationalökonomie und Statistik; Bd. 200, Nr. 4, S. 360ff.

Schlesinger, H., Weber, M., Ziebarth, G. (1993): Staatsverschuldung - ohne Ende? Zur Rationalität und Problematik des öffentlichen Kredits, Darmstadt

Schmid, J. (2000): Wie kann man von anderen Ländern lernen? Neuere Ansätze in der vergleichenden Arbeitsmarktpolitikforschung; in: IAW Mitteilungen, H. 3, S. 4 - 12

Schmid, J., Niketta, R. (Hrsg.) (1998): Wohlfahrtsstaat: Krise und Reform im Vergleich, Marburg

Schumpeter, J.A. (1918): Die Krise des Steuerstaates, Graz und Leipzig

Spahn, H.-P. (1981): Keynes in der heutigen Wirtschaftspolitik; in: Bombach, G. et al. (Hrsg.); Der Keynesianismus, Bd. I: Theorie und Praxis keynesianischer Wirtschaftspolitik, Berlin u.a., S. $211-292$

Streit, M. (2000): Theorie der Wirtschaftspolitik, Düsseldorf

Tiepelmann, K., Dick, G. (1995): Grundkurs Finanzwissenschaft, Hamburg

Urban, H.-J. (Hrsg.) (2000): Beschäftigungsbündnis oder Standortpakt? Hamburg

Arne Heise

New Politics

Integrative Wirtschaftspolitik für das 21. Jahrhundert

2001 - 235 Seiten - ca. DM 48,50 - ÖS 354 - SFR 44,50 - ISBN 3-89691-503-7

Jörg Stadlinger (Hrsg.)

Reichtum heute

Diskussion eines kontroversen Sachverhalts

2001 - ca. 300 Seiten - ca. DM 48,50 - ÖS 354 - SFR 44,50 - ISBN 3-89691-504-5

\section{WESTFÄLISCHES DAMPFBOOT}

Dorotheenstr. 26a · 48145 Münster · Tel. 0251/6086080 · Fax 0251/6086020 e-mail: info @ dampfboot-verlag.de ·http://www.dampfboot-verlag.de 